\title{
The Role of Nasal Surgery in the Treatment of OSA
}

\author{
Kenny P. Pang
}

Published online: 31 January 2013

(C) Springer Science+Business Media New York 2013

\begin{abstract}
Obstructive sleep apnea (OSA) is the collapse of the upper airway during sleep. The airway in the sleep state is very different from the airway in the awake state. The tissues that are collapsing very often can be identified and treated surgically by the current reconstructive techniques that are available. We know that it is not a "level" that obstructs, but a particular anatomy that is obstructing. The "palate level" can be due to large tonsils, thick redundant palate, long thick uvula, and/or collapsing lateral pharyngeal walls. Hence, it is not enough to say that the "level of the palate" is obstructing. Similarly, the nasal pathologies should also be described in detail, as in septal deviation, turbinate hypertrophy and/or nasal polyposis. Most sleep surgeons recognise that the treatment of the nose in OSA is pivotal, but not primary. This means that surgery to the nose as a single operative site has minimal effect on sleep apnea parameters, with low success rates. However, nasal surgery is of great importance in the surgical armamentarium of OSA due to its ability to facilitate other OSA treatments and maximize patient quality of life.
\end{abstract}

Keywords Nasal surgery - Sleep apnea $\cdot$ Nasal pathology

\section{Introduction}

Snoring is noisy breathing during sleep, which has been historically believed to be a social nuisance and can cause significant social stigma. Snoring is caused by the vibration of the structures in the oral cavity - the soft palate, uvula,

K. P. Pang ( $\square)$

Asia Sleep Centre, 290, Orchard Road, Paragon Unit 18-04, Singapore 238859, Singapore

e-mail: drkpang@gmail.com tonsils, base of tongue, epiglottis and pharyngeal walls. The vibration of these structures is due to a secondary effect of turbulent airflow within the upper airway, primarily caused by the air flowing through the nasal passages. The presence of snoring is believed to be a loud alarm to alert one to the possibility of a sleep disorder, which can range from simple snoring to upper airway resistance syndrome (UARS) and obstructive sleep apnea (OSA). This spectrum of disorders has been termed sleep disordered breathing (SDB).

Sleep disordered breathing is related to reduced airflow through the upper airway during sleep, due either to complete or partial upper airway obstruction or increased upper airway resistance. These includes simple snorers (snorers who have no daytime somnolence and with a normal apnea-hypopnea index), UARS (snorers who have daytime somnolence but have a normal apnea-hypopnea index), and OSA (snorers who are both tired and have an abnormal apnea-hypopnea index).

Obstructive sleep apnea arises from the collapse of the upper airway coupled with muscle tone relaxation during sleep. The nasal valve is the narrowest part of the upper airway which extends from the nasal orifices through the nasal cavity to the hypopharynx. It is well accepted that the upper airway accounts for two-thirds of the entire airway which extends down to the alveoli. Hence, simple widening of the nasal valve and the entire nasal passage would significantly contribute to a decrease in negative pressure at inspiration during sleep and contribute to improvement of OSA [1].

\section{Anatomic Review}

Simplistically, the anatomy of the upper airway is essentially a balance between the contents (soft tissues) and its 
container/box (skeletal framework). The soft tissues in the upper airway can be divided into the adipose tissues, muscle groups, and lymphoid tissues. The presence of adipose tissues surrounding the airway plays a significant role in SDB. This has both local and systemic effects on upper airway size, collapse, and ventilation. Locally, there are fat deposits present under the mucosal membranes as well as surrounding the various muscles in the neck. Adipose tissue in the palate, tonsillar fossa, and even in the pharyngeal walls may directly reduce airway caliber and thereby worsen upper airway obstruction. More importantly, obesity (by chest wall and abdominal fat) reduces lung volume. This reduction in lung volume decreases pharyngeal airway volume and increases pharyngeal collapse during sleep, especially when supine via a decrease in tracheal traction also known as tracheal tug [2]. Decreased lung volume also worsens hypoxemia in sleep which destabilizes breathing control mechanisms.

The three segments of the nasal cavity include the nasal valve, cavum, and nasopharynx. The majority of nasal resistance is associated with the nasal valve and close attention is paid to the nostrils, soft tissue aperture, skeletal aperture, inferior sinus turbinates, and, nasal septal deviation. The cavum may contribute to obstruction via nasal polyps, choncha bullosa or other structures. The nasopharynx and posterior choanae commonly contribute to nasal obstruction in children via adenoid hypertrophy, but may also contribute to abnormal resistance in adults.

Based on basic physics and the patho-physiological basis of airflow dynamics, the proper assessment of the nasal cavity and passage is of essence. In addition, the upper airway in the nose itself represents over $75 \%$ of the entire airway tract resistance, from the nasal cavity to the minute alveoli.

During inspiration, negative pressure is created within the intra-pleural space (e.g., negative $8 \mathrm{cmH}_{2} \mathrm{O}$ ) in order to distend the alveoli and to suck in or inhale air from the atmosphere into the lungs for gaseous exchange and oxygenation of the blood. This act of inhalation exerts a negative pressure on the entire upper airway, including the hypopharyngeal, retro-glossal and retro-palatal space. Hypothetically, if there were any form of upper airway blockage within the nasal passage (e.g., a deviated nasal septum, enlarged swollen turbinates, nasal polyps, etc.), the lungs would have to work "harder" in order to create a "more negative pressure" (e.g., negative $30 \mathrm{cmH}_{2} \mathrm{O}$ ), to inhale air from the atmosphere; this would ultimately result in a greater negative pressure on the hypopharyngeal, retroglossal and retro-palatal space, leading inevitably to collapse and obstruction of the hypopharyngeal upper airway [3•]. Hence, it is important to understand that without any form of obstruction in the nose, the airflow into the lungs through the nose would be laminar; however, with any form of nasal blockage, there would be turbulent airflow within the nasal cavity and passage, resulting in higher nasal resistance, poorer nasal breathing, and snoring with vibration of the palate (the first site of contact from the turbulent airflow). Therefore, it is reasonable to conclude that surgical correction of anatomical obstruction of the nasal passage alone does not cure OSA, it can significantly decrease the negative pressure within the hypopharyngeal region.

\section{Physiologic Review}

Although a small upper airway is a prerequisite for most patients with OSA and SDB, this alone will not result in OSA and SDB. Similarly, treating localized anatomic abnormalities may not reduce or adequately treat OSA. Alterations in physiology are important contributors to disease as well. In non-obstructed breathing, a smaller upper airway is compensated by increased muscle activity during both wake and sleep. In patients with OSA, however, with sleep onset, muscle tone and protective reflexes are reduced. Airway size is additionally reduced, with the assumption of the supine posture, which causes lung volume to decrease and venous blood volume to increase. Both reduce upper airway size and the combined effect is to decrease ventilation during sleep. Without activation of upper airway muscles, death would likely result. Fortunately, arousal reopens the airway but at the cost of fragmented sleep, hypoxemia, and a ventilatory overshoot. These contribute to the symptoms and morbidity of OSA and to the cyclic nature of OSA. Awareness of the physiologic contribution assists the surgeon in understanding the global philosophy described below.

\section{Surgical Planning (Pang-Woodson Surgical Protocol)}

In the surgical planning for a patient with OSA, the most crucial factor would be "selecting the correct procedure to perform on the correct patient." With this as the core philosophy in managing patients with OSA, upper airway evaluation of the anatomy is vital. The caveat is the fact that compared to a relatively static upper airway in normal or non-apneic individuals, the upper airway structure in patients with OSA is dynamic during both sleep and wakefulness. The physical examination during the awake state includes both static anatomic elements and dynamic physiologic compensation. During sleep, compensation becomes unstable in patients with snoring and OSA and the dynamic variability further increases and differs by sleep stage during sleep. Hence, one must attempt to examine the 
upper airway, keeping in mind its dynamic nature. The different types of examinations provide complementary insight while bearing in mind the economic cost and morbidity involved in each examination employed. There are some important basic fundamentals that the sleep physician and surgeon should remember:

1. Nasal Surgery in OSA is Pivotal but not Primary Treatment of the nose in OSA is crucial in its pathophysiology and in terms of improving airflow dynamics. In a multi-level surgical plan, the nose should be considered and its repair will significantly aid in the success rate of OSA surgery. Surgical correction of the nose depends on the anatomical abnormality; it can range from a septoplasty, submucous resection, inferior turbinate reduction, turbinoplasty, endoscopic sinus surgery, to balloon sinuplasty.

2. Palate Surgery is Reconstructive Surgery, not Ablative Surgery

The traditional uvulopalatopharyngoplasty (UPPP) surgery is not acceptable as treatment for OSA currently. Every patient has a different palate shape, size and configuration during sleep and during the collapse in OSA. The UPPP fails miserably if it is used for every single OSA patient. There are currently latest new methods in treating palate obstruction with far better results; for example, for patients with lateral pharyngeal wall collapse, the expansion sphincter pharyngoplasty has a much higher $82.6 \%$ success rate [4], the anterior palatoplasty has showed good reduction in snoring and sleep apnea in Pang et al. [5] long-term 3 year result studies.

3. OSA Surgery is not a Single UPPP Operation

It must be understood that there can be three major areas of obstruction, namely the nose, palate and tongue. These areas on their own have a huge varied reasons and anatomical sites of obstruction, for example, a deviated septum is different from gross nasal polyps, lateral pharyngeal wall collapse is different from antero-posterior collapse of the palatal area, and huge lingual tonsil would be vastly different from a huge retro-glossal tongue.

4. Tongue Surgery is Crucial in the OSA Surgery Armamentarium

Patients with tongue and/or palatal collapse noted on clinical airway evaluation and/or drug-induced sleep endoscopy should have some form of tongue procedure. Patients who fall in the Friedman clinical stage II and III, will benefit from a tongue procedure. This tongue procedure can be performed as a multi-level surgical procedure with/without nose surgery, and/or palate surgical variations.

Pang et al. showed that only $6.9 \%$ of patients with mild OSA had a $>50 \%$ collapse of the base of tongue region (during Muller's maneuver examination), as compared to $65.9 \%$ of patients with severe OSA [6]; therefore, patients with severe OSA have a ten-times higher incidence of base of tongue obstruction compared to patients with mild OSA $(P<0.00001)$. Hence, patients who suffer from severe OSA might also benefit from a tongue procedure.

The type of tongue surgery is dependent on the surgical expertise of the surgeon and the type of technologies available in the centre. Typically, patients with huge obstructing lingual tonsils should be offered a lingual tonsillectomy; while patients with a floppy epiglottis might need an epiglottopexy.

\section{The Role of Nasal Surgery in Sleep Apnea}

As the nose is the primary route of entry for air that will ultimately be inhaled into the lungs, maintaining, creating and providing a smooth laminar flow of air into the lower airway is crucial. The nose needs to be opened for patients who require nasal continuous positive airway pressure (CPAP) mask in order to splint and open their airway during sleep. Hence, the indications for nasal surgery in OSA are listed below:

1. Significant/Symptomatic Nasal Pathology

Reversible or reactive nasal pathologies (like inflammatory allergic rhino-sinusitis, nasal polyps, and/or infective sinusitis) are associated with sleep-disordered breathing [7]. It is known that patients with significant inflammatory nasal disease and symptoms are less compliant with CPAP and/or oral appliance as oral appliances rely on mouth closure to be effective which is only be possible if the nose is patent). General principles of surgery, combined with long-term medical therapy, should be instituted in patients with SDB. In addition, an awareness of options to maximize the effectiveness of positive airway pressure (e.g., appropriate humidification, adjunctive sprays, etc.) and avoid exacerbation of underlying mucosal disease is important.

2. Significant Structural/Anatomic Nasal Pathology

It is well accepted that nasal surgery for obstructive or functional nasal deficits should be performed when it would improve the patient's OSA and/or increases compliance of other OSA treatments [8]. The American Academy of Sleep Medicine Taskforce lists septoplasty, functional rhinoplasty and nasal valve surgery as potential procedures to consider in patients with OSA and snoring.

3. As Part of Multi-level Surgery Treatment Modality to Improve SDB

Nasal surgery is generally part of the surgical armamentarium used to treat sleep apnea, as part of a 
multi-level surgical plan. Nasal surgery facilitates other treatments for SDB, rather than being a treatment modality in and of itself. However in some instances, a significant treatment effect may be achieved in reduction of snoring, improvement in daytime symptoms, or even reduction in markers of OSA severity [e.g., apnea-hypopnea indices (AHI)] $[9,10]$.

4. Failed Tolerance/Efficacy of CPAP/Oral Appliance Use

Patients with OSA on CPAP or MAS with poor compliance due to a blocked nose and/or nasal pathology should be considered for nasal surgery in order to improve device compliance. Objective measurements of nasal resistance confirm that nasal obstruction not only decreases device tolerance but also increase treatment efficacy [11, 12]. Most authors concur that nasal resistance before the commencement of CPAP is a major risk factor for failure to accept the device as a treatment modality [13]. The average duration of CPAP use has also been shown to increase, and average CPAP pressure decrease, after upper airway interventions involving nasal surgery [14].

Nakata et al. [15] also showed that reducing nasal resistance correlates directly with improved quality of life and reduction in daytime sleepiness. Overall, it is crucial to note that nasal surgery reduces resistance and likely improves acceptance of CPAP, but does not significantly improve AHI [16].

\section{The Effect of Nasal Surgery on Obstructive Sleep Apnea}

Most authors would agree that the nasal surgery, as a single site procedure, would not significantly impact sleep apnea severity, but can have a significant effect on snoring. Verse et al. [17] showed through a meta-analysis of nine studies with 102 patients with OSA that the results of nasal surgery alone for these patients are at best $<20 \%$. Li et al. [18] had similar findings in their meta-analysis of 13 articles from 1999 to 2009. Two studies provided control groups and 11 articles $(84.6 \%)$ consisted of prospective non-controlled clinical trials (level II in evidence strength). The weighted mean apnea/hypopnea index measured by polysomnography in nine studies decreased from $35.2 \pm 22.6$ to $33.5 \pm 23.8$ event $/ \mathrm{h}$ after nasal surgery (overall, $P=0.69$ ). The pooled success rate of nasal surgery in treating OSA was $16.7 \%$. Epworth sleepiness scale scores in eight studies decreased from $10.6 \pm 3.9$ to $7.1 \pm 3.7$ (overall, $P<0.001$ ). However, nasal surgery for snoring assessed by individual questionnaires and visual analog scale is reported to result in significant improvement
$(P<0.05)$. In addition, the effects of nasal surgery on sleep apnea parameters are noted to be unpredictable and unreliable [19].

Interestingly, a single study done by Friedman et al. [20] looked at 49 patients with OSA and actually showed worsening of RDI in patients with mild OSA undergoing nasal surgery alone. This study showed that subjective nasal breathing improved in 49 (98\%) patients, and snoring decreased or disappeared in $17(34 \%)$; while the remaining $33(66 \%)$ patients did not notice any significant change in their snoring. Daytime energy levels increased in $39(78 \%)$ patients and remained unchanged or worsened in $11(22 \%)$. Review of the polysomnographic data shows that the group overall did not have significant changes in respiratory disturbance index (RDI) or lowest oxygen saturation levels $\left(\mathrm{LSaO}_{2}\right)$. CPAP levels required to correct OSA decreased after nasal surgery $(P<0.01)$. However, patients with mild OSA showed significant worsening in RDI $(P<0.05)$, whereas $\mathrm{LSaO}_{2}$ levels were improved in the group with moderate OSA $(P<0.05)$. In patients with severe OSA neither the RDI levels nor the $\mathrm{LSaO}_{2}$ changed, but CPAP levels required to alleviate the obstruction after surgery were reduced $(P<0.01)$. Victores et al. $[21 \bullet \bullet]$ showed that 24 patients with OSA who underwent nasal surgery alone did not have any significant change in drug induced sleep endoscopy findings pre-operatively compared to post-operatively. Nasal surgery alone did not improve apnea index, arousal index or apnea hypopnea index in 22 patients with OSA [22••].

\section{Conclusion}

When dealing with a patient with sleep apnea, it is not adequate to ascertain the severity of the disease with a sleep test alone; it is imperative to assess the patient's upper airway and evaluate the airflow dynamics from the point of entry (the nose) to the laryngeal inlet. With this thorough airway assessment, the surgeon would then be able to make an informed decision of what surgical plan is best for the patient, bearing in mind that the nasal surgery is pivotal in the entire surgical treatment of OSA.

Disclosure No potential conflict of interest was reported.

\section{References}

Papers of particular interest, published recently, have been highlighted as:

- Of importance

•• Of major importance 
1. Kohler M, Bloch KE, Stradling JR. The role of the nose in the pathogenesis of obstructive sleep apnea and snoring. Eur Respir J. 2007;30(6):1208-15.

2. Schwab RJ, Remmers JE, Kuna ST. Anatomy and physiology of upper airway obstruction. In: Kryger MH, Roth T, Dement WC, editors. Textbook of principles and practice of sleep medicine. 5th ed. St. Louis: Elsevier Saunders; 2011. p. 1153-71.

3. - Pang KP, Woodson BT. Current concepts in evaluation and surgical panning in OSA. In: Pang KP, Woodson BT, Rotenberg $\mathrm{B}$, editors. Textbook of advanced surgical technique in snoring and obstructive sleep apnea. 1st ed. Plural Publishing, San Diego; 2013. (In press). The chapter illustrates the need for nose surgery in OSA treatment.

4. Pang KP, Woodson BT. Expansion sphincter pharyngoplasty: a new technique for the treatment of obstructive sleep apnea. Otolaryngol Head Neck Surg. 2007;137(1):110-4.

5. Pang KP, Tan R, Puraviappan P, Terris DJ. Anterior palatoplasty for the treatment of OSA: 3-year results. Otolaryngol Head Neck Surg. 2009;141(2):253-6.

6. Pang KP, Terris DJ, Podolsky R. Severity of obstructive sleep apnea: correlation with clinical examination and patient perception. Otolaryngol Head Neck Surg. 2006;135(4):555-60.

7. Georgalas C. The Role of the nose in snoring and obstructive sleep apnea: an update. Eur Arch Otorhinolaryngol. 2011;268(9): 1365-73.

8. Epstein L, et al. Clinical guidelines for the evaluation, management, and long-term care of obstructive sleep apnea in adults. JCSM. 2009;15(3):263-76.

9. Kim ST, Choi JH, Jeon HG. Polysomnographic effects of nasal surgery for snoring and obstructive sleep apnea. Acta Otolaryngol. 2004;124(3):297-300.

10. Nakata S, Noda A, Yasuma F. Effects of nasal surgery on sleep quality in obstructive sleep apnea syndrome with nasal obstruction. Am J Rhinol. 2008;22(1):59-63.

11. Bican A, Kahraman A, Bora I, Kahrini R, Hakyemez B. What is the efficacy of nasal surgery in patients with obstructive sleep apnea? J Craniofac Surg. 2010;21(6):1801-6.

12. Zeng B, Ng A, Qian J, Petocz P, Darendelier H, Cistulli P. Influence of nasal resistance on oral appliance treatment outcome in obstructive sleep apnea. Sleep. 2008;31(4):543-7.
13. Sugiura T, Noda A, Nakata $S$, et al. Influence of nasal resistance on initial acceptance of continuous positive airway pressure in treatment for obstructive sleep apnea syndrome. Respiration. 2007;74:56-60.

14. Chandrashekariah R, Shaman Z, Auckley D. Impact of upper airway surgery on CPAP compliance in difficult-to-manage obstructive sleep apnea. Arch Otolaryngol Head Neck Surg. 2008;134(9):926-30.

15. Nakata S, Noda A, Yasuma F, et al. Effects of nasal surgery on sleep quality in obstructive sleep apnea syndrome with nasal obstruction. Am J Rhinol. 2008;22:59-63.

16. Nakata S, Noda A, Yagi H, et al. Nasal resistance for determinate factor of nasal surgery in CPAP failure patients with obstructive sleep apnea syndrome. Rhinology. 2005;43:296-9.

17. Verse T, Pirsig W. Impact of impaired nasal breathing on sleepdisordered breathing. Sleep Breath. 2003;7(2):63-76.

18. Li HY, Wang PC, Chen YP, et al. Critical appraisal and metaanalysis of nasal surgery for obstructive sleep apnea. Am J Rhinol Allergy. 2011;25(1):45-9.

19. Kim ST, Choi JH, Jeon HG, et al. Polysomnographic effects of nasal surgery for snoring and obstructive sleep apnea. Acta Otolaryngol. 2004;124(3):297-300.

20. Friedman M, Tanyeri H, Lim JW, et al. Effect of improved nasal breathing on obstructive sleep apnea. Otolaryngol Head Neck Surg. 2000;122(1):71-4.

21. • Victores AJ, Takashima M. Effects of nasal surgery on the upper airway: a drug-induced sleep endoscopy study. Laryngoscope. 2012;122(11):2606-10. Nasal surgery improves overall airway dynamics, as seen in drug induced sleep endoscopy.

22. • Choi JH, Kim EJ, Kim YS, et al. Effectiveness of nasal surgery alone on sleep quality, architecture, position, and sleep-disordered breathing in obstructive sleep apnea syndrome with nasal obstruction. Am J Rhinol Allergy. 2011;25(5):338-41. Nasal surgery does improve sleep architecture and sleep quality, but falls short in improving apnea indices. 